\title{
Qualidade do sono e sonolência diurna em estudantes universitários: prevalência e associação com determinantes sociais
}

\section{Sleep quality and daytime sleepiness in university students: prevalence and association with social determinants}

\author{
Mayonara Fabíola Silva Araújo' 이 | mayonarafabiola@gmail.com \\ Xaíze de Fátima de Medeiros Lopes' ${ }^{1}$ (1) xaizemedeiros@hotmail.com \\ Carolina Virginia Macedo de Azevedo² (1) carolina@cb.ufrn.br \\ Diego de Sousa Dantas ${ }^{3}$ (1) diegodantas1@gmail.com \\ Jane Carla de Souza' (1) jane.souza@ufrn.br
}

\begin{abstract}
RESUMO
Introdução: As alterações no ciclo sono/vigília (CSV) dos jovens universitários podem acarretar consequências na saúde física, psíquica e social. Além disso, alguns comportamentos adotados nessa fase podem estar associados a comprometimento do CSV.

Objetivo: Portanto, este estudo tem por objetivo identificar quais fatores dos determinantes sociais da saúde (DSS) estão associados à má qualidade do sono e à sonolência diurna excessiva (SDE) de universitários.

Método: Trata-se de um estudo transversal que incluiu 298 universitários, com idade entre 18 e 35 anos, 73,2\% dos estudantes do sexo feminino do interior do Rio Grande do Norte, Brasil. Os dados foram coletados a partir dos seguintes questionários: A Saúde e o Sono, Questionário de Cronotipo de Munique, Índice de Qualidade do Sono de Pittsburgh e Escala de Sonolência de Epworth. Para avaliar a associação dos DSS com a má qualidade de sono e a SDE, realizou-se a regressão de Poisson com variância robusta.

Resultado: A prevalência da má qualidade do sono e de SDE nos universitários foi de $79,2 \%$ e $51,3 \%$, respectivamente. Entre os determinantes intermediários da saúde, observou-se maior razão de prevalência de má qualidade de sono nos estudantes que apresentaram problema de saúde no último mês $(18,4 \%)$, fumavam $(23,5 \%)$ e faziam uso de bebidas estimulantes próximo ao horário de dormir $(25,8 \%)$, e naqueles que usavam eletrônicos antes do horário de dormir durante a semana (18,4\%), quando comparados aos que não adotam esses comportamentos. Com relação à SDE, os estudantes que justificaram o horário de dormir na semana e de acordar no fim de semana por causa da demanda acadêmica apresentaram $27 \%$ e $34 \%$, respectivamente, de menor prevalência de SDE do que o grupo que não adota esses comportamentos.
\end{abstract}

Conclusão: As altas prevalências de má qualidade do sono e de SDE observadas nos universitários foram decorrentes de fatores biológicos e, em sua maioria, de fatores comportamentais.

Palavras-chave: Sono; Determinantes Sociais da Saúde; Estudantes; Sonolência.

\begin{abstract}
Introduction: Changes in the Sleep/Wake Cycle (SWC) of university students can have consequences on physical, mental and social health. In addition, some behaviors adopted at this stage may be associated with SWC impairment.

Objective: Therefore, this study aims to identify which factors of social determinants of health (SDH) are associated with poor sleep quality and excessive daytime sleepiness (EDS) in university students.

Method: This is a cross-sectional study that included 298 university students, aged between 18 and 35 years; $73.2 \%$ of the students were females and from the countryside of the state of Rio Grande do Norte, Brazil. Data were collected from the following questionnaires: Health and Sleep, Munich Chronotype Questionnaire, Pittsburgh Sleep Quality Index and Epworth Sleepiness Scale. To assess the association of SDH with poor sleep quality and excessive daytime sleepiness, Poisson Regression with robust variance was performed.

Result: The prevalence of poor sleep quality and excessive daytime sleepiness among the university students was $79.2 \%$ and $51.3 \%$, respectively. Between the intermediate determinants of health, a higher prevalence rate of poor sleep quality was observed in students who reported health problems in the previous month (18.4\%), smoked (23.5\%), drank stimulating beverages close to bedtime (25.8\%) and those who used electronic devices before bedtime during the week (18.4\%) when compared to those who did not have these behaviors. Regarding excessive daytime sleepiness, students who justified bedtime during the week and wake-up time at the weekend because of the academic demand showed, respectively, 27\% and 34\% lower prevalence of EDS than the group that did not have these behaviors.
\end{abstract}

Conclusions: The high prevalence of poor sleep quality and EDS observed among university students was associated to biological factors and most of them, behavioral factors.

Keywords: Sleep; Social Determinants of Health; Students; Somnolence.

\footnotetext{
1 Universidade Federal do Rio Grande do Norte, Santa Cruz, Rio Grande do Norte, Brasil.

${ }^{2}$ Universidade Federal do Rio Grande do Norte, Natal, Rio Grande do Norte, Brasil.

${ }^{3}$ Universidade Federal de Pernambuco, Recife, Pernambuco, Brasil.
}

Editora-chefe: Daniela Chiesa

| Editor associado: Kristopherson Lustosa Augusto

Recebido em 03/06/20; Aceito em 05/04/21. | Avaliado pelo processo de double blind review. 


\section{INTRODUÇÃO}

A qualificação profissional é uma exigência crescente do mercado de trabalho da sociedade contemporânea, o que acarreta aumento de jovens buscando o ensino superior ${ }^{1}$. O Plano Nacional de Educação (PNE) - Lei no 10.172/2001 - teve como meta prover educação superior para no mínimo $30 \%$ da faixa etária de 18 a 24 anos $^{2}$. Em virtude disso, ocorreram a expansão e a interiorização das universidades brasileiras. No Rio Grande do Norte (RN), a universidade federal criou dez novos cursos presenciais de graduação no período de 2011 a 2015, entre os quais estão quatro cursos da Faculdade de Ciências da Saúde do Trairi, localizados no interior do estado 3 .

Entretanto, o ingresso no ensino superior é considerado uma experiência estressora, principalmente nos primeiros anos de graduação, momento em que ocorre a passagem da adolescência para a vida adulta ${ }^{4}$. Essa fase pode trazer repercussões para a vida dos estudantes, as quais envolvem delimitação da identidade pessoal, escolha profissional, transformação nas redes de amizade e apoio social, morar distante da família, alta cobrança de desempenho acadêmico e permanência no curso escolhido'.

A alta demanda acadêmica (DA) imposta aos universitários acarreta extensão da vigília ${ }^{5}$. Em contrapartida, os horários de início das aulas pela manhã obrigam os estudantes a acordar cedo, provocando privação de sono na semana e consequentemente aumento da sonolência diurna ${ }^{5}$.

O sono é fundamental para a manutenção da saúde e qualidade de vida ${ }^{6}$, e o bom desempenho acadêmico ${ }^{7}$. Contudo, para que as funções do sono sejam efetivas, é necessário manter diariamente uma quantidade e qualidade de sono, que varia ao longo da vida e de pessoa para pessoa. A duração de sono recomendada para jovens entre 18 e 25 anos é de sete a nove horas ${ }^{8}$.

Somado às dificuldades associadas às demandas acadêmicas, o sono dos jovens é afetado frequentemente por hábitos considerados não saudáveis, tais como: o consumo regular de substâncias psicoativas, como tabaco, álcool e café ${ }^{5}$, o sedentarismo ${ }^{6}$ e comportamentos alimentares inapropriados ${ }^{9}$.

Outro comportamento comum dos universitários é usar mídias à noite. $\mathrm{O}$ uso de smartphone após as 21 horas está relacionado a pior qualidade de sono, despertar mais tardio e mais tempo para iniciar o sono ${ }^{10}$. Esses dispositivos emitem luz artificial que atua no organismo causando redução da sonolência noturna, supressão da secreção de melatonina, atraso no horário de dormir e aumento da sonolência na manhã seguinte ao uso ${ }^{11}$. De acordo com Azevedo et al. ${ }^{12}$, o conhecimento sobre hábitos de higiene do sono pode ser um facilitador para a obtenção de um sono de boa qualidade, a percepção de bem-estar e a promoção da saúde dos indivíduos.
Vale ressaltar que a saúde do jovem universitário é resultado da interação entre as demandas inerentes ao ensino superior e os aspectos sociais, econômicos e pessoais, o que pode interferir de forma considerável na qualidade do sono e no nível de sonolência diurna desse público. Além disso, todos esses aspectos são considerados como indicadores de saúde populacional, os quais podem ser preditores de problemas crônicos de saúde, compondo os determinantes sociais da saúde (DSS).

De forma simples, a Organização Mundial da Saúde $(O M S)^{13}$ define os DSS como condições sociais em que as pessoas nascem, vivem, crescem, trabalham e envelhecem, tais como: habitação, saneamento, ambiente de trabalho, serviços de saúde e de educação, e estilos de vida individuais (tabagismo, alcoolismo, sedentarismo e hábitos alimentares).

Atualmente a OMS adota o modelo proposto por Solar et al. $^{14}$, o qual divide os determinantes essencialmente em determinantes estruturais das desigualdades (iniquidades) de saúde e determinantes intermediários. Os primeiros englobam fatores que geram estratificação social, tais como: distribuição de renda, preconceito com base no gênero, na etnia ou nas deficiências e estruturas políticas e de governança que aumentam iniquidades relativas ao poder econômico, gerando status socioeconômicos.

Além disso, os autores elencam como fatores contextuais desses determinantes as políticas macroeconômicas, como as fiscais e monetárias; as políticas nas áreas de emprego, posse de terra e habitação; as políticas públicas nas áreas da educação, saúde, água e saneamento, seguridade social e proteção social; a cultura e os valores sociais legitimados pela sociedade ${ }^{14}$.

Os determinantes intermediários são categorizados em circunstâncias materiais (condições de moradia, características da vizinhança, condições de trabalho, qualidade do ar, acesso a alimentos e água, e disponibilidade desses recursos), fatores comportamentais (estilo de vida e comportamentos), biológicos (fatores genéticos), psicossociais (estressores psicossociais, circunstâncias estressantes e falta de apoio social) e sistema de saúde ${ }^{14}$.

Considerando que houve um crescente aumento da população universitária decorrente do processo de interiorização e que existem poucos estudos sobre como o contexto de vida dos jovens no interior do estado interfere no sono, este estudo tem por objetivo identificar quais fatores dos DSS estão associados à má qualidade do sono e à sonolência diurna excessiva (SDE) em universitários do interior do estado.

\section{MÉTODOS}

Trata-se de um estudo observacional, de corte transversal com abordagem quantitativa, aprovado pelo Comitê de Ética em Pesquisa da Faculdade de Ciências da Saúde do Trairi - 
Certificado de Apresentação para Apreciação Ética (CAAE) nº 72209517.3.0000.5568. Participaram da pesquisa 298 estudantes de cursos da saúde de uma unidade acadêmica da Universidade Federal do Rio Grande do Norte localizada na cidade de Santa Cruz (35.797 habitantes/624,356 km²) a 117 km da capital ${ }^{15}$.

Recrutaram-se os participantes em sala de aula e pelas redes sociais, e a amostra foi realizada por conveniência. A coleta de dados ocorreu entre novembro de 2017 e junho de 2019, sendo excluídos os meses de recesso e férias escolares. Incluíram-se os estudantes maiores de idade, de ambos os sexos, que assinaram o Termo de Consentimento Livre e Esclarecido. Excluíram-se sete participantes que não preencheram completamente os questionários e um que apresentou idade destoante da média, sendo considerado um outlier.

Utilizaram-se quatro questionários autoaplicáveis:

- $\quad$ S Saúde e o Sono ${ }^{16}$ : utilizado amplamente em pesquisas no Brasil para avaliar hábitos de saúde e sono de uma população, incluindo 20 questões a respeito dos conhecimentos sobre sono. Acessa também informações como: sexo, idade, ter filhos, condições de moradia, percepção sobre o local de dormir, status socioeconômico, possuir problemas de saúde no último mês, praticar atividades físicas, razões relacionadas aos horários habituais de dormir e acordar, e hábitos realizados próximo ao horário de dormir.

- Questionário de Cronotipo de Munique ${ }^{17}$ : avalia horários habituais de dormir e acordar, e o período médio de sono durante os dias de estudo e livres. A partir dessas informações, é possível obter o cronotipo do indivíduo, que é definido pelo ponto médio do sono durante os dias livres corrigido (Midsleep Phase on Free Days sleep corrected - MSFsc), de 0 a 12 horas. Neste estudo, os indivíduos foram classificados em matutinos, intermediários ou vespertinos, de acordo com os critérios propostos por Roenneberg et al. ${ }^{18}$.

- $\quad$ Índice de Qualidade do Sono de Pittsburgh: avalia a qualidade do sono referente ao mês anterior a partir de sete componentes (qualidade subjetiva do sono, latência, duração e eficiência habitual do sono, distúrbios do sono, uso de medicamentos para dormir e disfunção diurna), e a pontuação de cada questão varia de 0 a 3, totalizando um escore final de 0 a 21 . Índices globais de 0 a 5 indicam uma boa qualidade do sono, e a partir de 6, uma má qualidade de sono ${ }^{19,20}$.

- Escala de Sonolência de Epworth: avalia a sonolência diurna por meio de oito situações cotidianas dos indivíduos em relação à propensão para cochilar. Cada situação é pontuada de 0 a 3 pontos, gerando um escore final entre 0 e 24. Pontuações até 10 são consideradas como indicativas de pouca sonolência diurna e maiores que 10 indicativas de SDE $^{21,22}$.

As variáveis estudadas foram distribuídas em dois grandes grupos de DSS: determinantes estruturais e intermediários. A alocação das variáveis nos grupos seguiu o modelo de DSS proposto por Solar et al. ${ }^{14}$. Dessa forma, a classificação econômica, categorizada em classes A, B, C e D-E pela Associação Brasileira de Empresas de Pesquisa (Abep) em 2018, o trabalho (se possui ou não atividade laboral), a escolaridade do chefe da família (analfabeto, ensino básico e ensino superior) e o nível de conhecimento, categorizado pela mediana de acertos ( $\leq 13$ acertos ou $\geq 14$ acertos), foram incluídos nos determinantes estruturais da saúde. O conhecimento foi avaliado a partir das 20 afirmativas sobre sono do questionário $\mathrm{A}$ Saúde e o Sono, com possibilidades de respostas de "verdadeiro", "falso" ou "não sei".

O segundo grupo foi de determinantes intermediários da saúde, agrupado em subgrupos de condições de moradia, fatores biológicos e fatores comportamentais: condição de moradia (morar ou não com a família) e local de dormir (bom ou ruim). Este último foi avaliado segundo a percepção do participante. Os fatores biológicos foram: sexo (masculino e feminino), cronotipo (matutino, intermediário e vespertino), apresentar problema de saúde no último mês (sim ou não) e idade, classificada de acordo com a faixa etária proposta nas recomendações da Fundação Americana do Sono, em que se consideram adultos jovens de 18 a 25 anos (indivíduos com idade $\leq 25$ anos ou $>25$ anos).

Os fatores comportamentais incluem variáveis relacionadas a hábitos gerais de vida e hábitos de sono realizados na hora de dormir ou até duas horas antes, apresentando respostas dicotômicas (sim ou não): praticar atividade física; fumar; usar bebidas estimulantes (café, chá preto ou mate, refrigerante do tipo cola e guaraná); usar bebidas alcoólicas; fazer alimentação inadequada antes de dormir (consumir uma refeição pesada ou dormir com fome); relatar DA antes de dormir ou como motivo para acordar; usar aparelhos eletrônicos antes de dormir; acordar para realizar afazeres domésticos, atividades recreativas e religiosas; relatar atividades de lazer antes de dormir.

A análise dos dados foi realizada por meio do software SPSS, versão 22. Calculou-se a prevalência da má qualidade do sono e da SDE. A regressão de Poisson, com variância robusta, forneceu a razão de prevalência (RP) e os respectivos intervalos de confiança (IC 95\%). As variáveis independentes que se associaram 
aos desfechos com $\mathrm{p}<0,20$, por meio da análise bivariada, foram incluídas nos modelos de regressão multivariados. Adotou-se como significância estatística um valor de $p<0,05$.

Reforça-se que a variável uso de eletrônico antes do horário de dormir durante a semana foi incluída no modelo final da regressão da qualidade de sono, pois apresenta valor de $\mathrm{p}=$ 0,06, e a literatura também aponta evidências da influência do uso de eletrônico na má qualidade do sono ${ }^{10,11}$.

\section{RESULTADOS}

A prevalência da má qualidade do sono e de SDE encontrada nos universitários foi de $79,2 \%$ e $51,3 \%$, respectivamente. A maioria dos estudantes é do sexo feminino (73,2\%), apresenta idade menor que 25 anos (90,6\%), não mora com a família $(87,2 \%)$, se deslocou para morar na cidade em que estuda (82,3\%), pertence às classes econômicas C $(52,3 \%)$ e B $(31,9 \%)$, não trabalha $(91,6 \%)$, possui chefe da família que estudou até o ensino básico (62,1\%), apresenta (52,3\%) menor nível de conhecimento sobre sono ( $<13$ acertos) e avalia como bom o local em que dorme $(91,9 \%)$.

Além disso, a maioria apresentou características de cronotipo vespertino (94,3\%), não teve problema de saúde no último mês $(56,7 \%)$ e relatou realizar algum tipo de atividade física (52\%), não fumar (98\%) nem consumir bebidas estimulantes (80,5\%), não usar bebidas alcoólicas (93,6\%) e não realizar alimentação inadequada (64,1\%).

Quanto aos hábitos relacionados ao ciclo sono/vigília, a maioria dos universitários alegou ter DA e fazer uso de eletrônicos (UE) antes de dormir durante a semana (DA = 76,2\%;
UE $=76,2 \%$ ) e fim de semana (DA $=69,5$; UE $=86,9 \%)$. A DA também apareceu como motivo de acordar durante a semana $(83,9 \%)$ e no fim de semana $(76,8 \%)$.

As atividades de lazer $(A L)$ e os afazeres domésticos (AD) antes do horário de dormir foram relatados pela minoria na semana ( $A L=26,8 \% ; A D=20,8 \%$ ), respectivamente. Contudo, $75,8 \%$ e $28,5 \%$ dos universitários relataram que realizam $A D$ e $A L$ antes de dormirem no fim de semana, respectivamente. Ademais, $A D$ não foram relatados pela maioria como motivo para acordar na semana $(79,5 \%)$ e no fim de semana $(68,8 \%)$.

Com relação aos determinantes estruturais da saúde, não foi observada nenhuma associação com a má qualidade do sono e a SDE dos universitários (Tabela 1).

As tabelas 2 e 3 mostram as variáveis classificadas como determinantes intermediários da saúde que apresentaram $\mathrm{p}<$ 0,20 na análise bivariada da associação com a má qualidade do sono e a SDE, as quais foram incluídas nos modelos de regressão multivariados.

Dentre as variáveis associadas à má qualidade do sono, o modelo multivariado da regressão demonstrou uma prevalência 18,4\% maior ( $\mathrm{RP}=1,18$ IC 95\% 1,06-1,32) de má qualidade do sono nos estudantes que apresentaram problema de saúde no último mês. Além disso, aqueles que fumam ( $R P=1,23$ IC 95\% 1,12-1,36), fazem uso de bebidas estimulantes ( $R P=1,26$ IC 95\% 1,14-1,38) e usam eletrônicos $(\mathrm{RP}=1,19$ IC 95\% 1,00-1,40) antes do horário de dormir apresentaram, respectivamente, 23,5\%, 26\% e 19\% maior prevalência de má qualidade de sono em relação aos jovens que não adotam esses comportamentos (Tabela 4).

Tabela 1. Análise bivariada dos determinantes estruturais de saúde que podem influenciar na má qualidade do sono e na sonolência diurna excessiva (SDE) dos universitários - Santa Cruz/RN, Brasil, 2020.

\begin{tabular}{|c|c|c|c|c|c|c|c|c|c|}
\hline \multirow{3}{*}{ Variáveis } & \multirow{3}{*}{ Categorias } & \multicolumn{2}{|c|}{$\begin{array}{c}\text { Má qualidade do sono } \\
\mathrm{n}(\%)\end{array}$} & \multicolumn{2}{|c|}{ Não ajustada } & \multicolumn{2}{|c|}{$\begin{array}{l}\text { SDE } \\
\mathrm{n}(\%)\end{array}$} & \multicolumn{2}{|c|}{ Não ajustada } \\
\hline & & SIM & NÃO & \multirow{2}{*}{ RP (IC 95\%) } & \multirow{2}{*}{ p valor } & SIM & NÃO & \multirow{2}{*}{ RP (IC 95\%) } & \multirow{2}{*}{ p valor } \\
\hline & & $236(79,2)$ & $62(20,8)$ & & & $154(51,3)$ & $145(48,7)$ & & \\
\hline \multirow{4}{*}{ Classe econômica } & Classe A & $8(72,7)$ & $3(27,3)$ & \multirow{4}{*}{$\begin{array}{c}0,98(0,81-1,06) \\
1\end{array}$} & \multirow{4}{*}{0,591} & $7(63,6)$ & $4(36,4)$ & \multirow{4}{*}{$\begin{array}{c}0,99(0,85-1,16) \\
1\end{array}$} & \multirow{4}{*}{0,947} \\
\hline & Classe B & $77(81,1)$ & $18(18,9)$ & & & $46(48,4)$ & $49(51,6)$ & & \\
\hline & Classe C & $125(80,1)$ & $31(19,9)$ & & & $82(52,6)$ & $74(47,4)$ & & \\
\hline & Classe D-E & $26(72,2)$ & $10(27,8)$ & & & $18(50)$ & $18(50)$ & & \\
\hline \multirow{2}{*}{ Trabalha } & Sim & $20(80)$ & $5(20)$ & \multirow{2}{*}{$\begin{array}{c}1,01(0,82-1,24) \\
1\end{array}$} & \multirow{2}{*}{0,916} & $9(36)$ & $16(64)$ & \multirow{2}{*}{$\begin{array}{c}0,68(0,40-1,16) \\
1\end{array}$} & \multirow{2}{*}{0,361} \\
\hline & Não & $216(79,1)$ & $57(20,9)$ & & & $144(52,7)$ & $129(47,3)$ & & \\
\hline \multirow{3}{*}{$\begin{array}{l}\text { Escolaridade do } \\
\text { chefe da família }\end{array}$} & Analfabeto & $36(76,6)$ & $11(23,4)$ & \multirow{3}{*}{$\begin{array}{c}1,01(0,92-1,11) \\
1\end{array}$} & \multirow{3}{*}{0,829} & $26(55,3)$ & $21(44,7)$ & \multirow{3}{*}{$\begin{array}{c}1,00(0,84-1,20) \\
1\end{array}$} & \multirow{3}{*}{0,963} \\
\hline & Nível básico & $148(80)$ & $37(20)$ & & & $91(49,2)$ & $94(50,8)$ & & \\
\hline & Nível superior & $52(78,8)$ & $14(21,5)$ & & & $36(54,5)$ & $30(45,5)$ & & \\
\hline \multirow{2}{*}{$\begin{array}{l}\text { Conhecimento } \\
\text { sobre o sono }\end{array}$} & $\leq 13$ acertos & $128(82,1)$ & $28(17,9)$ & \multirow{2}{*}{$\begin{array}{c}1,08(0,96-1,21) \\
1\end{array}$} & \multirow{2}{*}{0,207} & $80(51,3)$ & $76(48,7)$ & \multirow{2}{*}{$\begin{array}{c}0,99(0,80-1,24) \\
1\end{array}$} & \multirow{2}{*}{0,983} \\
\hline & $\geq 14$ acertos & $108(76,1)$ & $34(23,9)$ & & & $73(51,4)$ & $69(48,6)$ & & \\
\hline
\end{tabular}

RP: razão de prevalência; IC: intervalo de confiança. 
Tabela 2. Análise bivariada das variáveis de condições de moradia, fatores biológicos e comportamentais classificados como determinantes intermediários da saúde que podem influenciar na má qualidade do sono e na sonolência diurna excessiva (SDE) dos universitários - Santa Cruz/RN, Brasil, 2020.

\begin{tabular}{|c|c|c|c|c|c|c|c|c|c|}
\hline \multirow{3}{*}{ Variáveis } & \multirow{3}{*}{ Categorias } & \multicolumn{2}{|c|}{$\begin{array}{c}\text { Má qualidade do sono } \\
\mathrm{n}(\%)\end{array}$} & \multicolumn{2}{|c|}{ Não ajustada } & \multicolumn{2}{|c|}{$\begin{array}{l}\text { SDE } \\
\mathrm{n}(\%)\end{array}$} & \multicolumn{2}{|c|}{ Não ajustada } \\
\hline & & SIM & NÃO & \multirow{2}{*}{ RP (IC 95\%) } & \multirow{2}{*}{ p valor } & SIM & NÃO & \multirow{2}{*}{ RP (IC 95\%) } & \multirow{2}{*}{$p$ valor } \\
\hline & & $236(79,2)$ & $62(20,8)$ & & & $154(51,3)$ & $145(48,7)$ & & \\
\hline \multirow{2}{*}{ Moradia: com a família } & $\operatorname{Sim}$ & $29(76,3)$ & $9(23,7)$ & $1,04(0,86-1,26)$ & \multirow{2}{*}{0,658} & $16(42,1)$ & $22(57,9)$ & $1,25(0,85-1,85)$ & \multirow{2}{*}{0,260} \\
\hline & Não & $207(79,6)$ & $53(20,4)$ & 1 & & $137(52,7)$ & $123(47,3)$ & 1 & \\
\hline \multirow{2}{*}{ Local de dormir } & Bom & $216(78,8)$ & $58(21,2)$ & $1,06(0,87-1,28)$ & \multirow{2}{*}{0,565} & $141(51,5)$ & $133(48,5)$ & $0,97(0,64-1,47)$ & \multirow{2}{*}{0,892} \\
\hline & Ruim & $20(83,3)$ & $4(16,7)$ & 1 & & $12(50)$ & $12(50)$ & 1 & \\
\hline \multirow{2}{*}{ Sexo } & Masculino & $63(78,8)$ & $17(21,2)$ & $1,01(0,89-1,15)$ & \multirow{2}{*}{0,909} & $38(47,5)$ & $42(52,5)$ & $1,11(0,85-1,44)$ & \multirow{2}{*}{0,433} \\
\hline & Feminino & $173(79,4)$ & $45(20,6)$ & 1 & & $115(52,8)$ & $103(47,2)$ & 1 & \\
\hline \multirow{3}{*}{ Cronotipo } & Matutino & $2(100)$ & $0(0)$ & \multirow{3}{*}{$\begin{array}{c}0,95(0,80-1,12) \\
1\end{array}$} & \multirow{3}{*}{0,527} & $2(100)$ & $0(0)$ & \multirow{3}{*}{$\begin{array}{c}1,07(0,68-1,698) \\
1\end{array}$} & \multirow{3}{*}{0,764} \\
\hline & Intermediário & $12(80)$ & $3(20)$ & & & $5(33,3)$ & $10(66,7)$ & & \\
\hline & Vespertino & $222(79)$ & $59(21)$ & & & $146(52)$ & $135(48)$ & & \\
\hline \multirow{2}{*}{$\begin{array}{l}\text { Problema de saúde/ } \\
\text { último mês }\end{array}$} & Sim & $113(87,6)$ & $16(12,4)$ & $1,20(1,07-1,35)$ & \multirow{2}{*}{$<0,001$} & $71(55)$ & $58(45)$ & $1,13(0,91-1,41)$ & \multirow{2}{*}{0,262} \\
\hline & Não & $123(72,8)$ & $46(27,2)$ & 1 & & $82(48,5)$ & $87(51,5)$ & 1 & \\
\hline \multirow{2}{*}{ Idade } & $\leq 25$ anos & $217(80,4)$ & $53(19,6)$ & $1,18(0,91-1,54)$ & \multirow{2}{*}{0,205} & $140(51,9)$ & $130(48,1)$ & $1,12(0,74-1,70)$ & \multirow{2}{*}{0,601} \\
\hline & $>25$ anos & $19(67,9)$ & $9(32,1)$ & 1 & & $13(46,4)$ & $15(53,6)$ & 1 & \\
\hline \multirow{2}{*}{ Atividade física } & Sim & $123(79,4)$ & $32(20,6)$ & $0,94(0,89-1,12)$ & 0 & $84(54,2)$ & $71(46,8)$ & $0,89(0,71-1,11)$ & 0 \\
\hline & Não & $113(79)$ & $30(21)$ & 1 & 0,94 & $69(48,3)$ & $74(51,7)$ & 1 & , \\
\hline Háhi & Sim & $6(100)$ & $0(0)$ & $1,27(1,20-1,35)$ & & $4(66,7)$ & $3(33,3)$ & $1,31(0,73-2,33)$ & \\
\hline & Não & $230(78,8)$ & $62(21,1)$ & 1 & & $149(51)$ & $143(49)$ & 1 & \\
\hline Uso de bebidas & & & & & & & & & \\
\hline Ectimulanto & Sim & $55(94,8)$ & $3(5,2)$ & $1,26(1,14-1,38)$ & ( 000 & $28(48,3)$ & $30(51,7)$ & $0,93(0,692-1,242)$ & 0611 \\
\hline Estimulante & Não & $181(75,4)$ & $59(24,6)$ & 1 & $<0,001$ & $125(52,1)$ & $115(47,9)$ & 1 & 0,011 \\
\hline Alcoólicas & Sim & $18(94,7)$ & $1(5,3)$ & $1,21(1,07-1,37)$ & בחחת - - 20 & $9(47,4)$ & $10(52,6)$ & $0,92(0,56-1,49)$ & 0730 \\
\hline & Não & $218(78,1)$ & $61(21,9)$ & 1 & 0,002 & $144(51,6)$ & $135(48,4)$ & 1 & 0 \\
\hline Alimentacão inadequada & Sim & $86(80,4)$ & $21(19,6)$ & $1,02(0,91-1,15)$ & 0704 & $55(51,4)$ & $52(48,6)$ & $1,00(0,80-1,26)$ & 0988 \\
\hline 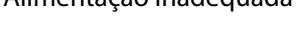 & Não & $150(78,5)$ & $41(21,5)$ & 1 & 0,704 & $98(51,3)$ & $93(48,7)$ & 1 & 0,700 \\
\hline
\end{tabular}

RP: razão de prevalência; IC: intervalo de confiança.

No modelo de regressão multivariado, foi observado que aqueles que relataram dormir ( $\mathrm{RP}=0,66 \mathrm{IC} 95 \% 0,48-$ $0,91)$ por causa da DA nos dias da semana e acordar ( $\mathrm{RP}=0,73$ IC 95\% 0,54-0,98) pelo mesmo motivo nos fins de semana apresentaram uma prevalência de $27 \%$ e $34 \%$ menor de SDE do que o grupo que não adotava esse comportamento (Tabela 5).

\section{DISCUSSÃO}

Este estudo teve por objetivo identificar a prevalência e os fatores associados à má qualidade do sono e à SDE em universitários do interior do estado do $\mathrm{RN}$, a partir do modelo de Solar et al. ${ }^{14}$ de DSS.

$\mathrm{Na}$ análise dos determinantes estruturais da saúde, nenhuma variável apresentou associação com a má qualidade do sono e a SDE dos universitários estudados. Apesar disso, este estudo apresenta contribuição pioneira para a discussão do impacto de DSS no sono de universitários, tendo em vista que a utilização de um modelo de DSS que inclui inúmeras variáveis minimiza os possíveis vieses de trabalhos que avaliam separadamente fatores relacionados à saúde do sono. Ressaltase ainda que os dados foram obtidos a partir de questionários validados e amplamente utilizados em pesquisa.

A prevalência de má qualidade do sono observada neste estudo foi de $79,2 \%$, similar a outros estudos $(72,2 \%-81,5 \%)$ realizados com universitários de capitais ${ }^{23,24}$. Vale ressaltar que grandes cidades apresentam características culturais e econômicas diferenciadas em relação a municípios do interior. Além das altas demandas acadêmicas, os universitários das grandes cidades possuem estilo de vida com enorme influência da poluição luminosa noturna e das grandes distâncias de deslocamentos, maior acesso à atividade de lazer, entre outros fatores que impactam negativamente o sono ${ }^{25}$. 
Tabela 3. Análise bivariada dos hábitos relacionados aos horários de sono e vigília na semana e no fim de semana classificados como determinantes intermediários da saúde que podem influenciar na má qualidade do sono e na sonolência diurna excessiva (SDE) dos universitários - Santa Cruz/RN, Brasil, 2020.

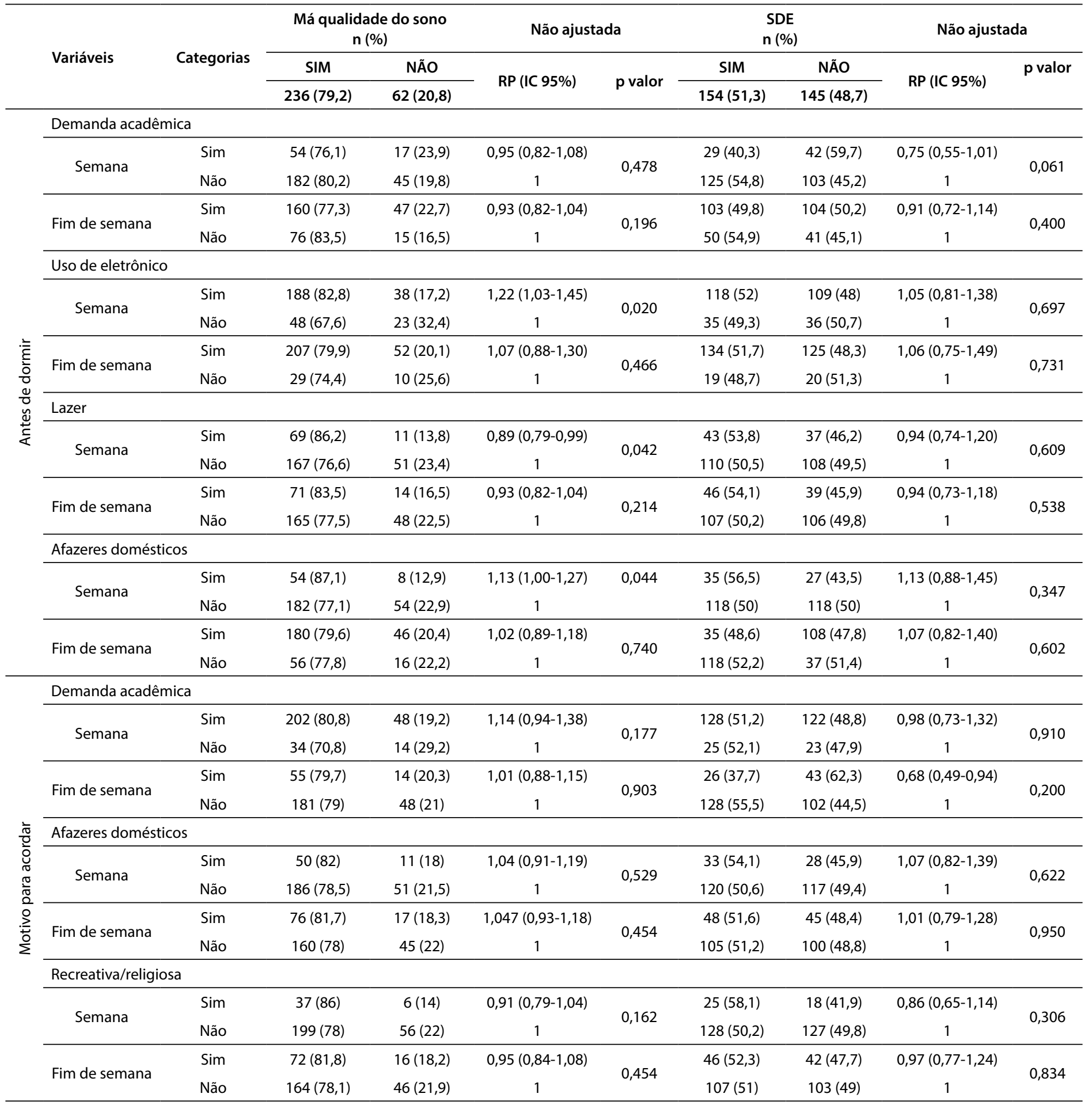

RP: razão de prevalência; IC: intervalo de confiança. 
Tabela 4. Análise multivariada dos aspectos que influenciam na má qualidade do sono dos universitários - Santa Cruz/RN, Brasil, 2020.

\begin{tabular}{|c|c|c|c|c|c|c|}
\hline \multirow{2}{*}{\multicolumn{2}{|c|}{ Variáveis }} & \multirow{2}{*}{ Categorias } & \multicolumn{2}{|c|}{ Não ajustada } & \multicolumn{2}{|c|}{ Ajustada } \\
\hline & & & RP (IC 95\%) & p valor & RP (IC 95\%) & p valor \\
\hline \multirow{2}{*}{\multicolumn{2}{|c|}{ Problemas de saúde no último mês }} & Sim & $1,17(1,05-1,31)$ & \multirow{2}{*}{$0,004^{*}$} & $1,18(1,06-1,32)$ & \multirow{2}{*}{$0,003^{*}$} \\
\hline & & Não & 1 & & 1 & \\
\hline \multirow{2}{*}{\multicolumn{2}{|c|}{ Hábito de fumar }} & $\operatorname{Sim}$ & $1,22(1,06-1,39)$ & \multirow{2}{*}{$0,005^{*}$} & $1,23(1,12-1,36)$ & \multirow{2}{*}{$<0,001^{*}$} \\
\hline & & Não & 1 & & 1 & \\
\hline \multirow{16}{*}{ 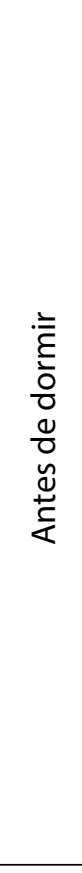 } & Uso de bebidas & & & & & \\
\hline & \multirow{2}{*}{ Estimulantes } & Sim & $1,12(1,07-1,33)$ & \multirow{2}{*}{$0,001^{*}$} & $1,26(1,14-1,38)$ & \multirow{2}{*}{$<0,001^{*}$} \\
\hline & & Não & 1 & & 1 & \\
\hline & \multirow{2}{*}{ Alcoólicas } & Sim & $1,08(0,94-1,24)$ & \multirow{2}{*}{0,278} & & \multirow{2}{*}{-} \\
\hline & & Não & 1 & & - & \\
\hline & \multirow{2}{*}{ Uso de eletrônicos } & Sim & $1,17(0,99-1,38)$ & \multirow{2}{*}{0,066} & $1,19(1,00-1,40)$ & \multirow{2}{*}{$0,046^{*}$} \\
\hline & & Não & 1 & & 1 & \\
\hline & \multicolumn{6}{|l|}{ Demanda acadêmica } \\
\hline & \multirow{2}{*}{ Fim de semana } & Sim & $0,95(0,85-1,06)$ & \multirow{2}{*}{0,381} & \multirow{2}{*}{ - } & \multirow{2}{*}{-} \\
\hline & & Não & 1 & & & \\
\hline & \multicolumn{6}{|l|}{ Afazeres domésticos } \\
\hline & \multirow{2}{*}{ Semana } & Sim & $1,07(0,95-1,20)$ & \multirow{2}{*}{0,243} & \multirow{2}{*}{-} & \multirow{2}{*}{-} \\
\hline & & Não & 1 & & & \\
\hline & \multicolumn{6}{|l|}{ Lazer } \\
\hline & \multirow{2}{*}{ Semana } & Sim & $0,96(0,86-1,08)$ & 0551 & & \\
\hline & & Não & 1 & ונכבו & 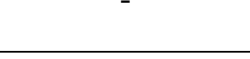 & 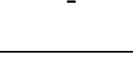 \\
\hline & Demanda acadêmica & & & & - & - \\
\hline$\frac{0}{0}$ & Semana & Sim & $1,11(0,92-1,33)$ & 0.264 & - & - \\
\hline$\gg \frac{\pi}{0}$ & ectiranta & Não & 1 & $0, \angle 07$ & & \\
\hline$\underbrace{\mathcal{E}}_{0} \mathcal{U}_{0}$ & Recreativa/religiosa & & & & & \\
\hline$\stackrel{\xi}{\varrho}$ & Semana & Sim & $0,92(0,791,06)$ & 0.233 & - & - \\
\hline & & Não & 1 & 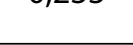 & & \\
\hline
\end{tabular}

RP: razão de prevalência; IC: intervalo de confiança. *Valor significativo $(p<0,05)$.

Parâmetros do modelo: deviance, $\mathrm{p}=0,357$; omnibus test, $\mathrm{p}=0,231$; subdispersão dos dados $\left(X^{2}, \mathrm{p}=0,211\right)$.

Tabela 5. Análise multivariada dos aspectos que influenciam na sonolência diurna excessiva (SDE) dos universitários - Santa Cruz/ RN, Brasil, 2020.

\begin{tabular}{|c|c|c|c|c|c|}
\hline \multirow{2}{*}{ Variáveis } & \multirow{2}{*}{ Categorias } & \multirow{2}{*}{$\begin{array}{c}\text { Não ajustada } \\
\text { RP (IC 95\%) }\end{array}$} & \multicolumn{3}{|c|}{ Ajustada } \\
\hline & & & $p$ valor & RP (IC 95\%) & $p$ valor \\
\hline \multicolumn{6}{|l|}{ Antes de dormir } \\
\hline \multirow{2}{*}{ Semana } & Sim & $0,75(0,55-1,01)$ & \multirow{2}{*}{0,061} & $0,66(0,48-0,91)$ & \multirow{2}{*}{$0,035^{*}$} \\
\hline & Não & 1 & & 1 & \\
\hline \multicolumn{6}{|l|}{ Demanda acadêmica } \\
\hline \multirow{2}{*}{ Fim de semana } & Sim & $0,68(0,49-0,94)$ & \multirow{2}{*}{0,020} & $0,73(0,54-0,98)$ & \multirow{2}{*}{$0,012^{*}$} \\
\hline & Não & 1 & & 1 & \\
\hline
\end{tabular}

RP: razão de prevalência; IC: intervalo de confiança. *Valor significativo $(p<0,05)$.

Parâmetros do modelo: deviance, $p=0,677)$; omnibus test, $p=0,048$; subdispersão dos dados $\left(X^{2}, p=0,490\right)$. 
Em contrapartida, este estudo foi realizado com universitários de um município no qual a população mantém hábitos interioranos, pois a maioria do comércio fecha no horário do almoço e funciona até o início da noite, não tendo hipermercados ou outros serviços de comércio 24 horas. As opções de lazer são reduzidas a poucos restaurantes e lanchonetes, não existindo shopping centers, cinemas, teatros ou boates, e comumente as pessoas se locomovem a pé ou usam o serviço de mototáxi, pois não há grandes distâncias de deslocamento. Portanto, faltam diversificação das atividades econômicas e oferta de serviços em relação à capital do estado.

Diante disso, os resultados observados neste estudo destacam que as questões referentes à má qualidade do sono, que é frequente em universitários das grandes cidades, são atualmente problemas de saúde pública que atingem também universitários de municípios do interior. Isso pode ser decorrente da expansão de uma sociedade que consome, trabalha e socializa 24 horas por dia, sete dias na semana (sociedade 24/7), sobretudo por meio das tecnologias ${ }^{26}$. Assim, independentemente da localidade geográfica, a alta prevalência de má qualidade do sono em universitários pode ser decorrente da rotina de afazeres, demanda acadêmica, socialização e estímulos digitais.

No modelo de regressão multivariado, foi possível observar que alguns hábitos de vida e ter problemas de saúde foram fatores associados à má qualidade de sono. Contudo, as prevalências observadas foram baixas quando comparadas ao estudo de Martinez et al. ${ }^{27}$. Apesar disso, deve ser considerado o impacto do consumo de bebidas estimulantes na fisiologia do sono, o que aumenta a atividade simpática e a interrupção do sono ${ }^{27}$, refletindo na má qualidade do sono. $\mathrm{O}$ hábito de fumar associado à má qualidade de sono foi também observado em universitários de Fortaleza, no Ceará7, o que pode estar associado a distúrbios na arquitetura do sono, provocando um sono mais leve, maior latência no início do sono e diminuição da eficiência do sono ${ }^{28}$.

Ressalta-se que a associação da má qualidade de sono ao hábito de fumar observada neste estudo pode ser avaliada como uma correlação não verdadeira, tendo em vista que apenas seis indivíduos relataram esse hábito. No entanto, destacamos que o teste estatístico adotado não apresenta requisito de razão de proporção de amostra, como estabelecido em testes de comparação de médias. A regressão de Poisson, com estimador robusto, é considerada o método mais adequado para estimar as razões de prevalência em estudos transversais, seja de efeitos comuns (> 10\%) ou não ${ }^{29}$. Diante disso, podemos inferir que o estilo de vida desses universitários de municípios do interior do estado que culturalmente apresentam hábitos mais saudáveis e com menor acesso à exposição de substância psicoativa pode ter contribuído para o resultado observado.
De forma geral, os problemas de saúde impactam a qualidade do sono, que, por sua vez, compromete a recuperação da saúde do indivíduo. Vale ressaltar que o sono é considerado fundamental para a saúde humana, devido à sua atividade reparadora com grande influência no funcionamento dos sistemas nervoso e cardíaco ${ }^{30}$, na restauração do metabolismo energético, no fortalecimento do sistema imunológico, na sensação de bem-estar, entre outros ${ }^{31}$.

Além disso, foi observado que os universitários que relataram fazer "uso de eletrônicos (TV, computador, smartphone) antes do horário de dormir" apresentaram pior qualidade do sono. $\mathrm{O}$ uso desses aparelhos próximo ao horário de dormir pode afetar negativamente o sono, pois o estímulo luminoso suprime a melatonina, hormônio indutor de sono em humanos ${ }^{32}$, e aumenta o alerta em consequência da maior atividade do sistema nervoso, resultando em dificuldade para adormecer $^{10}$, o que pode ser ainda maior se o tipo de conteúdo acessado tiver teor violento, provocativo ou perturbador ${ }^{32}$.

Com relação à $\mathrm{SDE}$, a prevalência observada foi de $51,3 \%$, índice considerado maior em relação aos resultados obtidos em outros estudos (36,8\% e 32,2\%) que foram realizados com público de mesma faixa etária e utilizando o mesmo instrumento de avaliação ${ }^{33,34}$. Esses estudos também observaram maior prevalência de SDE nas mulheres em relação aos homens. Portanto, considerando que nossa amostra foi prioritariamente de mulheres, isso pode ter contribuído para a alta prevalência observada. No entanto, a literatura aponta o sexo feminino relacionado a pouca queixa de sonolência diurna, porém pode ser que essa associação se estabeleça apenas em faixas etárias mais avançadas ${ }^{35}$.

Somada a esses fatores, a SDE pode estar associada às altas demandas acadêmicas, às aulas que acontecem muito cedo pela manhã, ao uso de aparelhos eletrônicos antes de dormir $^{10,11}$, à privação de sono e aos horários irregulares de dormir e acordar ${ }^{10,11}$, o que demonstra que a qualidade do sono e a sonolência diurna apresentam influência multifatorial ${ }^{36-38}$.

Entre os determinantes intermediários da saúde, observou-se que a realização de DA para acordar no fim de semana e antes de dormir na semana foi associada a pouca sonolência diurna. Esses comportamentos podem estar contribuindo para a manutenção de uma regularidade nos horários de sono, a qual está relacionada à menor sonolência diurna observada ${ }^{34}$. De acordo com a literatura, os estudantes tendem a acordar mais cedo na semana, devido ao horário das aulas, e dormir e acordar, espontaneamente, mais tarde no fim de semana ${ }^{33,34}$.

Além disso, essas atividades acadêmicas são realizadas sob a influência da luz natural ou artificial, a qual gera alerta fisiológico e pode provocar maior resistência ou adaptação 
fisiológica ao indivíduo, de modo a gerar um viés de percepção do estudante ao preencherem a escala de avaliação da sonolência diurna. Ademais, há diferenças individuais com relação à necessidade biológica da duração de sono ${ }^{39}$. Nesse sentido, mesmo que os estudantes estejam realizando DA antes dos horários de dormir e para acordar, o que poderia acarretar privação de sono e SDE, isso pode não está acontecendo, pois os estudantes podem ser pequenos dormidores e estar cumprindo com a necessidade biológica de sono.

É importante destacar que a associação entre DA e menor sonolência poderia estar relacionada aos hábitos sociais matutinos vivenciados em cidades do interior, de maior alerta ao acordar $^{40}$. Contudo, essa influência do cronotipo matutino foi considerada no estudo e não apresentou associação significativa com a sonolência diurna.

Assim, a prevalência da má qualidade do sono e da SDE nos universitários aqui estudados é decorrente da condição de saúde do indivíduo e, na sua maioria dos casos, está relacionada a aspectos comportamentais. Esses resultados são de suma importância para discussões e planejamento das aulas e atividades extracurriculares (estudos, estágios e atividades complementares), pois é imprescindível que haja uma flexibilização de horários de estudo para promover melhor adaptação aos horários de sono dos estudantes.

Como limitações deste estudo, ressalta-se que a análise de determinantes estruturais da saúde pode ter sido comprometida, pois eles englobam fatores contextuais complexos e amplos, tais como: políticas públicas de educação, habitação, macroeconômicas e outras. Diante da complexidade de os determinantes estruturais da saúde e a classificação socioeconômica da Abep se referirem à situação da família e não à da moradia a que o estudante está exposto, nossos resultados podem ter sido comprometidos, uma vez que $82,3 \%$ dos participantes avaliados neste estudo se deslocaram para morar na cidade do campus universitário, apresentando condições de moradia diferente do contexto familiar.

Além disso, destaca-se que $52 \%$ dos universitários deste estudo não atingiram a pontuação mínima de conhecimentos sobre hábitos adequados de sono. Isso demonstra que a importância do sono para a saúde é possivelmente pouco discutida na formação desses profissionais. Contudo, conhecer aspectos sobre a higiene do sono não necessariamente acarreta a prática de hábitos adequados, visto que os estudantes podem estar expostos a altas demandas acadêmicas, que são geralmente realizadas à noite.

Espera-se que este estudo sirva de subsídio para fomentar discussões no âmbito universitário que resultem em melhoria na qualidade do sono e diminuição da SDE, proporcionando qualidade de vida, saúde e melhores condições de aprendizagem aos jovens universitários, e na implantação de programas educacionais sobre o sono, que podem contribuir para a elaboração de políticas públicas, particularmente no contexto da interiorização do ensino.

Além disso, os estudantes abordados são profissionais da saúde em formação, e essas discussões podem ajudar a ter profissionais mais sensíveis e capazes de prevenir os distúrbios de sono, identificá-los e intervir neles, gerando impactos positivos nos âmbitos social e de saúde populacional.

\section{CONCLUSÕES}

Os universitários do interior do estado do $\mathrm{RN}$ apresentaram alta prevalência de má qualidade de sono, e metade da amostra teve SDE. Dentre os DSS, a maior prevalência de má qualidade do sono foi associada a fatores considerados determinantes intermediários da saúde, tais como: apresentar problema de saúde no último mês, ser fumante, fazer uso de bebidas estimulantes e utilizar aparelhos eletrônicos antes de dormir.

Com relação à $S D E$, os casos de universitários que relataram realizar DA antes dos horários de dormir na semana e como motivo para acordar no fim de semana foram associados a pouca sonolência diurna. Entre os determinantes estruturais da saúde, nenhuma associação foi observada com a má qualidade de sono e a SDE.

Torna-se necessário que estudos futuros ampliem a avaliação de outros fatores que podem causar interferência na qualidade do sono e na SDE nesse público, tais como início dos horários de aula, duração do sono e irregularidade nos horários de dormir e acordar.

\section{CONTRIBUIÇÃO DOS AUTORES}

Mayonara Fabíola Silva Araújo e Jane Carla de Souza participaram da concepção e do design do estudo, da aquisição, análise e interpretação dos dados, e da redação, revisão e aprovação final da versão a ser publicada. Xaíze de Fátima de Medeiros Lopes participou da concepção e do design do estudo, e da aquisição, análise e interpretação dos dados. Carolina Virginia Macedo de Azevedo participou da concepção e do design do estudo, e da redação e revisão do artigo. Diego de Sousa Dantas participou da concepção e do design do estudo, e da revisão do artigo.

\section{CONFLITO DE INTERESSES}

Declaramos não haver conflito de interesses.

\section{FINANCIAMENTO}

Declaramos não haver financiamento. 


\section{REFERÊNCIAS}

1. Oliveira CT, Santos AS, Dias ACG. Expectativas de universitários sobre a universidade: Sugestões para facilitar a adaptação acadêmica. Rev Bras Orientaç Prof. 2016;17(1):43-53.

2. Brasil. Lei no 10.172 , de 9 de janeiro de 2001. Plano Nacional de Educação (PNE). Brasília; 2001 [access in 22 apr 2019]. Available from: http://www. planalto.gov.br/ccivil_03/leis/leis_2001/110172.htm.

3. Universidade Federal do Rio Grande do Norte. Plano Diretor do Campus de Santa Cruz. Resolucão no 073/2017-CONSAD 2017 [access in 24 apr 2019]. Available from: https://ufrn.br/institucional/documentos.

4. Teixeira MAP, Dias ACG, Wottrich SH, Oliveira AM. Adaptação à universidade em jovens calouros. Psicol Esc e Educ. 2008;12(1):185-202.

5. Lopes HS, Meier DAP, Rodrigues R. Qualidade do sono entre estudantes de enfermagem e fatores associados. Ciências Biológicas e da Saúde. 2018;39(2):129-36 [access in 24 apr 2019]. Available from: http://www.uel. br/revistas/uel/index.php/seminabio/article/view/32236/24760.

6. Andrade RD, Felden ÉPG, Teixeira CS, Pelegrinii A. Sono, percepção de saúde e atividade física em adolescentes universitários. Adolescência \& Saúde. 2017;14(4):150-6.

7. Araújo MFM, Freitas RWJF, Lima ACS, Pereira DCR, Zanetti ML, Damasceno MMC. Health indicators associated with poor sleep quality among university students. Rev Esc Enferm USP. 2014;48(6):1082-9 [access in 11 apr 2019]. Available from: http://www.scielo.br/scielo.php?pid=S008062342014000601085\&script=sci_arttext\&tlng=pt.

8. Hirshkowitz M, Whiton K, Albert SM, Alessi C, Bruni O, DonCarlos L, et al. National sleep foundation's sleep time duration recommendations: methodology and results summary. Sleep Heal. 2015;1(1):40-3. doi: 10.1016/j.sleh.2014.12.010.

9. Perez PMP, Castro IRR, Franco AS, Bandoni DH, Wolkoff DB. Práticas alimentares de estudantes cotistas e não cotistas de uma universidade pública Brasileira. Cienc Saude Colet. 2016;21(2):531-42.

10. Amra B, Shahsavari A, Shayan RM, Mirheli O, Moradi BK, Bazukar M, et al. Associação entre o sono e o uso noturno de celular entre adolescentes. J Pediatr (Rio J). 2017;93(6):560-7. doi: 10.1016/j.jped.2016.12.004.

11. Chang AM, Aeschbach D, Duffy JF, Czeisler CA. Evening use of lightemitting eReaders negatively affects sleep, circadian timing, and nextmorning alertness. Proc Natl Acad Sci U S A. 2015;112(4):1232-7.

12. Azevedo CVM, Sousa I, Paul K, Macleish MY, Mondéjar MT, Sarabia JA, et al. Teaching chronobiology and sleep habits in school and university. Mind, Brain, Educ. 2008;2(1):34-47.

13. Organização Mundial da Saúde. Redução das desigualdades no período de uma geração. Igualdade na saúde através da acção sobre os seus determinantes sociais. Relatório final da comissão para os determinantes sociais da saúde. OMS; 2010 [access in 12 may 2019]. Available from: http://www.who.int/eportuguese/publications/pt/.

14. Solar O, Irwin A. A conceptual framework for action on the social determinants of health. Soc Determ Heal Discuss Pap 2 (Policy Pract). Discussion Paper Series on Social Determinants of Health 2010;79 [access in 01 feb 2019]. Available from: http://apps.who.int/iris/ bitstream/10665/44489/1/9789241500852_eng.pdf?ua=1\&ua=1.

15. Instituto Brasileiro de Georgrafia e Estatística. Cidades@. IBGE; 2010 [access in 12 may 2019]. Available from: https://cidades.ibge.gov.br/ brasil/rn/santa-cruz/panorama.

16. Mathias $A$, Sanchez $R$, Andrade $M$. Incentivar hábitos de sono adequados: um desafio para os educadores. In: Pinho S, Sagliett IJ, editores. Núcleos de Ensino da Unesp. São Paulo; 2006. p. 718-31.

17. Levandovski R, Sasso E, Hidalgo MP. Chronotype: a review of the advances, limits and applicability of the main instruments used in the literature to assess human phenotype. Trends Psychiatry Psychother. 2013;35(1):3-11.

18. Roenneberg T, Wirz-Justice A, Merrow M. Life between clocks: daily temporal patterns of human chronotypes. J Biol Rhythms. 2003;18(1):8090. doi: $10.1177 / 0748730402239679$.

19. Buysse DJ, Reynolds CF, Monk TH, Berman SR, Kupfer DJ. The Pittsburgh Sleep Quality Index: a new instrument for psychiatric practice and research. Psychiatry Res. 1989:28:193-213 [access in 23 feb 2019]. Available from: https://www.sciencedirect.com/science/article/ pii/0165178189900474?via\%3Dihub.
20. Bertolazi AN, Fagondes SC, Hoff LS, Dartora EG, Miozzo ICS, Barba MEF, et al. Validation of the Brazilian Portuguese version of the Pittsburgh Sleep Quality Index. Sleep Med. 2011;12(1):70-5. doi: 10.1016/j.sleep.2010.04.020.

21. Bertolazi AN, Fagondes SC, Hoff LS, Pedro VD, Barreto SSM, Johns MW. Validação da Escala de Sonolência de Epworth em português para uso no Brasil. J Bras Pneumol. 2009;35(9):877-83 [access in 23 feb 2019]. Available from: http://www.scielo.br/scielo.php?script=sci_arttext\&pid=S180637132009000900009\&lng=en\&nrm=iso\&tlng=pt.

22. Johns MW. A new method for measuring daytime sleepiness: the Epworth sleepiness scale. Sleep. 1991;14(6):540-5.

23. Segundo LVG, Cavalcanti Neto BF, Paz DDA, Holanda MMA. Aspectos relacionados à qualidade do sono em estudantes de medicina. Rev Bras Neurol Psiquiatr. 2017;21(3):213-23.

24. Carvalho TMCS, Silva II, Siqueira PPSS, Almeida JO, Soares AF, Lima AMJ. Qualidade do sono e sonolência diurna entre estudantes universitários de diferentes áreas. Rev Neurociênc. 2013;21(3):383-7.

25. Pilz LK, Levandovski R, Oliveira MAB, Hidalgo MP, Roenneberg T. Sleep and light exposure across different levels of urbanisation in Brazilian communities. Sci Rep. 2018;8(1):1-11.

26. Bezerra BB. Sociedade de consumo e o universo 24/7. MATRIZes. 2017:11(1):213-6.

27. Martinez G, Escãno $\mathrm{HC}$, Sousa $\mathrm{MH}$, Pinto CAL. Impacto do etanol e consumo de café na qualidade de sono de acadêmicos de medicina. Rev Med (São Paulo). 2018;97(3):267-72 [access in 19 apr 2019]. Available from: https://www.revistas.usp.br/revistadc/article/view/144015/141864.

28. Jaehne A, Loessl B, Bárkai $Z$, Riemann $D$, Hornyak M. Effects of nicotine on sleep during consumption, withdrawal and replacement therapy. Sleep Med Rev. 2009;13(5):363-77 [access in 11 apr 2019]. Available from: https://www.sciencedirect.com/science/article/pii/ S1087079208001329?via\%3Dihub.

29. Legler U, Roback P. Chapter 4. Poisson regression. In: Broadening your statistical horizons: generalized linear models and multilevel models. Florida Ed CRC press. 2019. p. 440 - 454 [access in 11 may 2020]. Available from https://bookdown.org/roback/bookdown-BeyondMLR/ch-poissonreg.html.

30. Jansen J, Lopes A, Jansen U, Capone D, Maeda T, Noronha A, et al. Medicina da noite: da cronobiologia à prática clínica. 2008. p. 340

31. Guimar SS. Impacto dos transtornos do sono sobre o funcionamento diário e a qualidade de vida. Estud Psicol. 2007;24(4):519-28.

32. Owens J, Maxim R, McGuinn M, Nobile C, Msall M, Alario A. Televisionviewing habits and sleep disturbance in school children. Pediatrics. 1999; 104(3) p.01-08.

33. Barbosa SMML, Batista RFL, Rodrigues LDS, Bragança MLBM, Oliveira BR Simões VMF, et al. Prevalência de sonolência diurna excessiva e fatores associados em adolescentes da coorte RPS, em São Luís (MA). Rev Bras Epidemiol. 2020;23:e200071.

34. Carone CMM, Silva BDP, Rodrigues LT, Tavares PDS, Carpena MX, Santos IS Factors associated with sleep disorders in university students. Cad Saude Publica. 2020;36(3):1-16.

35. Silva A, Andersen ML, Mello MT, Bittencourt LRA, Peruzzo D, Tufik $S$. Gender and age differences in polysomnography findings and sleep complaints of patients referred to a sleep laboratory. Brazilian J Med Bio Res. 2008:41(12):1067-5.

36. Bittencourt LRA, Silva RS, Santos RF, Pires MLN, Mello MT. Excessive daytime sleepiness. Encycl Neurol Sci. 2014;27(Suppl I):233-5.

37. Winwood PC, Lushington K. Disentangling the effects of psychological and physical work demands on sleep, recovery and maladaptive chronic stress outcomes within a large sample of Australian nurses. J Adv Nurs. 2006;56(6):679-89 [access in 13 may 2019] . Available from: https://www. ncbi.nlm.nih.gov/pubmed/17118046.

38. Pereira ÉF, Teixeira CS, Louzada FM. Sonolência diurna excessiva em adolescentes:prevalênciaefatoresassociados.RevPaulPediatr.2010;28(1):98 103 [access in 23 apr 2021]. Available from: http://pepsic.bvsalud.org/scielo. php?script=sci arttext\&pid=S1415-88092013000200002.

39. Webb WB. Are short and long sleepers different? Psychol Rep. 1979;44:25964. doi: 10.2466/pro.1979.44.1.259.

40. Duarte LL. Cronotipos humanos. Cruz das Almas, BA: Editora UFRB; 2018 $108 \mathrm{p}$

This is an Open Access article distributed under the terms of the Creative Commons Attribution License, which permits unrestricted use, distribution, and reproduction in any medium, provided the original work is properly cited. 